\title{
Extended Kalman Filter for Oversampled Dynamical Phase Offset Estimation
}

\author{
Jordi Vilà Valls, Student Member, IEEE, Jean-Marc Brossier and Laurent Ros \\ GIPSA-lab, Departement Image Signal \\ BP 46 - 38402 Saint Martin d'Hères - FRANCE \\ E-mail: jordi.vilavalls@gipsa-lab.inpg.fr, jean-marc.brossier@gipsa-lab.inpg.fr, laurent.ros@gipsa-lab.inpg.fr
}

\begin{abstract}
In this paper, we present an application of the Extended Kalman Filter for the on-line estimation of a dynamical carrier phase offset. The novel approach implies deriving the filter in an oversampled scenario in a digital receiver. We consider a Brownian phase evolution in a Data Aided scenario. Our numerical results using a BOC shaping pulse show that using the oversampled signal for estimating the phase offset we can obtain better performances than using a classical synchronizer.

Index Terms-Phase estimation, Extended Kalman Filter, oversampling, carrier synchronization, GALILEO, BOC.
\end{abstract}

\section{INTRODUCTION}

Synchronization is a fundamental part in modern digital receivers. A synchronizer has to estimate some parameters, such as carrier frequency, carrier phase and timing epoch. This knowledge is required to recover the signal of interest correctly. In this paper we focus our attention on the phase estimation problem. Many methods for estimating the phase introduced by an unknown channel have been proposed over the past decades, from Phase Locked Loops (PLL) to the most sophisticated signal processing techniques. The Kalman Filter (KF) [3], [4], presented in early 1960s is one of the mostly used techniques for parameter estimation in linear gaussian problems. We can find extensive discussion on the KF in [5] and [6]. When dealing with nonlinear filtering problems, the Extended Kalman Filter (EKF) approximates the problem to apply the KF solution. Some contributions show the use of EKF for carrier phase recovery and frequency tracking [9][12]. To our knowledge, the EKF has never been applied to oversampled phase estimation for Binary Offset Carrier (BOC) shaped signals.

In this contribution, we investigate the use of an Extended Kalman Filter for carrier estimation in a Data Aided (DA) scenario. We consider an oversampled signal model after receiver matched filter, this implies having a coloured reception noise. This scenario is standard in radio-localization from a satellite signal. In [7], we have shown the potential gain for phase estimation provided by the use of the fractionaly-spaced signal after matched filter, instead of the symbol time-spaced signal. This was done by deriving a closed-form expression of the on-line Bayesian Cramér-Rao Bound (BCRB) for the oversampled dynamical phase estimation. Now, our goal is to propose an EKF based algorithm which can approach this bound. We have thus to jointly estimate the coloured noise and the phase offset because the EKF doesn't take it into account. In Section II, we set the signal model. In Section III, we derive the expressions of the filter in the oversampled phase estimation scenario. In Section IV we recall the BCRB for this estimation problem. Finally in Section V, the numerical results for the EKF resulting from BPSK transmission are presented and interpreted.

Notations: italic indicates a scalar quantity, as in $a$; boldface indicates a vector quantity, as in a and capital boldface indicates a matrix quantity as in $\mathbf{A}$. The $(k, l)^{t h}$ entry of a matrix $\mathbf{A}$ is denoted $[\mathbf{A}]_{k, l}$. The matrix transpose and selfadjoint operators are denoted by the superscripts ${ }^{T}$ and $H$ respectively as in $\mathbf{A}^{T}$ and $\mathbf{A}^{H} . \Re(\cdot), \Im(\cdot)$ and $(\cdot)^{*}$ are the real part, the imaginary part and conjugate of a complex number or matrix, respectively. $\mathrm{E}_{x}$ denotes the expectation over $x$.

\section{SignAl MOdeL}

We propose the signal model for the transmission of a known sequence $\left\{a_{m}\right\}_{m \in \mathbb{Z}}$ over an Additive White Gaussian Noise (AWGN) channel affected by a carrier phase offset $\theta(t)$.

\section{A. Oversampled Signal Model}

1) Discrete-time general formulation: the received complex baseband signal after matched filtering is

$$
y(t)=\left[\left\{T \sum_{m} a_{m} \Pi(t-m T)\right\} e^{i \theta(t)}+n(t)\right] * \Pi^{*}(-t)
$$

where $T, \Pi(t)$ and $n(t)$ stands for the symbol period, shaping pulse and circular gaussian noise with a known bilateral power spectral density (psd) $N_{0}$.

We define the filtered noise

$$
b(t)=[n(t)] * \Pi^{*}(-t)
$$

We also define $\tilde{g}_{m}(t)$ as

$$
\tilde{g}_{m}(t)=T \int_{-\infty}^{+\infty} \Pi^{*}(-\alpha) e^{i \theta(t-\alpha+m T)} \Pi(t-\alpha) d \alpha
$$

Then the received signal can be written as

$$
y(t)=\sum_{m} a_{m} \tilde{g}_{m}(t-m T)+b(t)
$$


Hereafter we suppose a shaping pulse with support in $[0, T]$ and a slow varying phase evolution during a period $T$. In this case we can approximate $\tilde{g}_{m}(t)$ by

$$
\tilde{g}_{m}(t) \approx g(t) e^{i \theta\left(t+\left(m+\frac{1}{2}\right) T\right)}
$$

where

$$
g(t)=T \int_{-T}^{0} \Pi^{*}(-\alpha) \Pi(t-\alpha) d \alpha
$$

If the received signal is fractionally-spaced at $t_{k}=k \frac{T}{S}+\tau$, where $S$ is an integer oversampling factor and $\tau$ a known offset from the optimum sampling instants (we suppose $0 \leq \tau \leq \frac{T}{S}$ ), we have that

$$
y\left(k \frac{T}{S}+\tau\right)=\sum_{m} a_{m} \tilde{g}_{m}\left(k \frac{T}{S}+\tau-m T\right)+b\left(k \frac{T}{S}+\tau\right)
$$

and from (eq. 5) we have that

$$
y\left(k \frac{T}{S}+\tau\right)=e^{i \theta\left(k \frac{T}{S}+\tau+\frac{T}{2}\right)} A_{k}+b\left(k \frac{T}{S}+\tau\right)
$$

where

$$
A_{k}=\sum_{m} a_{m} g\left(k \frac{T}{S}+\tau-m T\right) .
$$

We can finally write the received oversampled signal as

$$
y_{k}=A_{k} e^{i \theta_{k}}+b_{k}^{\prime}
$$

where $k$ refers to $t_{k}$ instants.

We can define the symbol index $p=\left\lfloor\frac{k}{S}\right\rfloor$, or equivalently, $k=p S+s$ with $s$ the sample inside the symbol period. We note that $s=0, \cdots, S-1$.

Note that the noise $b_{k}^{\prime}$ is coloured with variance $\sigma_{n}^{2}$, where $\sigma_{n}^{2}=N_{0} \times \frac{g(0)}{T}$ is the variance of the AWGN noise $n(t)$ measured in the noise equivalent bandwidth of the receiver filter $\Pi^{*}(-t)$.

2) Discrete-time re-formulation for the noise: the $\frac{T}{S}$-spaced sequence of noise, $\left\{b_{k}^{\prime}\right\}_{k \in \mathbb{Z}}$, is defined in the previous section from an analog noise $n(t)$. Our motivation now is to replace this time serie by another $\left\{b_{k}\right\}_{k \in \mathbb{Z}}$ with the same statistical properties, but which can be obtained entirely by a discretetime formulation. This will be useful for the final state-space model formulation. We can write that

$$
\begin{aligned}
b_{k}^{\prime} & =\int_{0}^{T} n\left(\alpha+k \frac{T}{S}+\tau\right) \Pi^{*}(\alpha) \mathrm{d} \alpha \\
& =\sum_{j=0}^{S-1} \int_{j \frac{T}{S}}^{(j+1) \frac{T}{S}} n\left(\alpha+k \frac{T}{S}+\tau\right) \Pi^{*}(\alpha) \mathrm{d} \alpha
\end{aligned}
$$

We define $\boldsymbol{\Gamma}$ as the covariance matrix of the observation noise. If we have $N$ measurements the matrix is $N \times N$ and depends on the oversampling factor $S$.

The random variables

$$
Z_{k, j}=\int_{j \frac{T}{S}}^{(j+1) \frac{T}{S}} n\left(\alpha+k \frac{T}{S}+\tau\right) \Pi^{*}(\alpha) \mathrm{d} \alpha
$$

are zero-mean gaussian distributed. For a fixed $k, Z_{k, j}$ are independent in $j$. Their variance is equal to

$$
\mathrm{E}\left(\left|Z_{k, j}\right|^{2}\right)=N_{0} \int_{j \frac{T}{S}}^{(j+1) \frac{T}{S}}|\Pi(\alpha)|^{2} \mathrm{~d} \alpha
$$

We define a zero-mean, unit variance, gaussian i.i.d sequence $n_{k}$ and

$$
\Pi_{j}=\left\{N_{0} \int_{j \frac{T}{S}}^{(j+1) \frac{T}{S}}|\Pi(\alpha)|^{2} \mathrm{~d} \alpha\right\}^{\frac{1}{2}}
$$

Hence, the noise samples $b_{k}^{\prime}$ have the same statistical properties than samples $b_{k}$ obtained by a $\frac{T}{S}$-spaced filtering of the time serie $n_{k}$ :

$$
b_{k}=\sum_{j=0}^{S-1} \Pi_{j} n_{k-j-1}
$$

\section{B. Phase-offset Evolution Model}

In practice we must consider jitters introduced by clocks imperfections. To take it into account we consider a Brownian phase-offset evolution [13]

$$
\theta_{k}=\theta_{k-1}+w_{k} \quad k \geq 2
$$

where $w_{k}$ is an i.i.d. zero-mean Gaussian noise with known variance $\frac{\sigma_{w}^{2}}{S}$. Here $\sigma_{w}^{2}$ stands for the variance growth of the phase noise in one symbol period. The $N \times N$ covariance matrix of the phase-offset evolution is $\boldsymbol{\Sigma}$.

\section{State-Space Model}

When using an optimal filtering approach a state-space model formulation is needed. Moreover, as we want to take into account that the observation noise is not white, we must include it into the state evolution.

We consider a sliding vector $\left[\begin{array}{llll}\nu_{k} & \nu_{k-1} & \cdots & \nu_{k-S+1}\end{array}\right]^{T}$ over an i.i.d. noise $n_{k}$, the evolution of this vector can be written as

$\left[\begin{array}{c}\nu_{k} \\ \nu_{k-1} \\ \vdots \\ \nu_{k-S+1}\end{array}\right]=\left[\begin{array}{cccc}0 & \cdots & \cdots & 0 \\ 1 & 0 & \cdots & 0 \\ 0 & \ddots & & \vdots \\ & & 1 & 0\end{array}\right]\left[\begin{array}{c}\nu_{k-1} \\ \nu_{k-2} \\ \vdots \\ \nu_{k-S}\end{array}\right]+\left[\begin{array}{c}n_{k} \\ 0 \\ \vdots \\ 0\end{array}\right]$

The coloured noise $b_{k}$ is

$$
b_{k}=\left[\Pi_{0} \cdots \Pi_{S-1}\right]\left[\begin{array}{c}
\nu_{k-1} \\
\nu_{k-2} \\
\vdots \\
\nu_{k-S}
\end{array}\right]
$$


The state to be considered includes the phase-offset and the coloured noise: $\mathbf{x}_{k}=\left[\begin{array}{lllll}\theta_{k} & b_{k} & \nu_{k} & \cdots & \nu_{k-S+1}\end{array}\right]^{T}$. The state evolution is

$$
\mathbf{x}_{k}=\mathbf{M} \mathbf{x}_{k-1}+\mathbf{w}_{k}
$$

where

$$
\mathbf{M}=\left[\begin{array}{cccccc}
1 & 0 & 0 & \ldots & & 0 \\
0 & 0 & \Pi_{0} & & & \Pi_{S-1} \\
0 & 0 & 0 & 0 & \ldots & 0 \\
\vdots & \vdots & 1 & & & \\
& & & \ddots & & \\
0 & 0 & \cdots & 0 & 1 & 0
\end{array}\right]
$$

and

$$
\mathbf{w}_{k}=\left[\begin{array}{llllll}
w_{k} & 0 & n_{k} & 0 & \cdots & 0
\end{array}\right]^{T}
$$

The observation equation can be written as

$$
\begin{aligned}
y_{k} & =A_{k} \exp \left(i\left[\begin{array}{llll}
1 & 0 & \cdots & 0
\end{array}\right]^{T} \mathbf{x}_{k}\right) \\
& +\left[\begin{array}{lllll}
0 & 1 & 0 & \cdots & 0
\end{array}\right]^{T} \mathbf{x}_{k}
\end{aligned}
$$

We note that the state equation is linear and the observation equation depends non-linearly on the state. With this formulation we have no observation noise because we have included it in the state.

\section{EXTENDED KALMAN FILTER}

In the sequel we introduce the well known EKF. We can find the general EKF expressions in [5]. Then we derive the expressions on the oversampled carrier phase estimation scenario.

We have a system described by the following state-space equations pair

$$
\begin{aligned}
& \mathbf{x}_{k+1}=f_{k}\left(\mathbf{x}_{k}\right)+\mathbf{w}_{k} \\
& \mathbf{y}_{k}=g_{k}\left(\mathbf{x}_{k}\right)+\mathbf{v}_{k}
\end{aligned}
$$

where $\mathbf{x}_{k}$ is the state vector, $\mathbf{w}_{k}$ is a zero-mean white noise with covariance matrix $\mathbf{Q}_{k}, \mathbf{y}_{k}$ is the observation vector at time $k$ which is a partial and noisy observation of the state $\mathbf{x}_{k}$ and $\mathbf{v}_{k}$ is the observation noise with covariance matrix $\mathbf{R}_{k}$

Both noises $\mathbf{w}_{k}$ and $\mathbf{v}_{k}$ are supposed to be uncorrelated. The functions $f_{k}(\cdot)$ and $g_{k}(\cdot)$ can be non-linear in a general case.

For Gaussian, linear state models, the KF gives the best Mean Square Error (MSE) estimation of the state $\mathbf{x}_{k}$ from observations up to time $k$.

We note $\widehat{\mathbf{x}}_{k \mid m}$, the estimation of $\mathbf{x}_{k}$ from the observations up to time $m, \widetilde{\mathbf{x}}_{k \mid m}=\mathbf{x}_{k}-\widehat{\mathbf{x}}_{k \mid m}$, the estimation error and $\mathbf{P}_{k \mid m}=$ $\mathrm{E}\left(\widetilde{\mathbf{x}}_{k \mid m} \widetilde{\mathbf{x}}_{k \mid m}^{T}\right)$, the covariance matrix of the estimation error.

The EKF gives us the estimator $\widehat{\mathbf{x}}_{k \mid k}$ in a recursive way. The main idea in the EKF is to linearize the state-space equations at each iteration in order to transform the filtering problem into a usual Kalman one.

So we need to compute

$$
\frac{\partial f_{k}\left(\mathbf{x}_{k}\right)}{\partial \mathbf{x}_{k}} ; \frac{\partial g_{k}\left(\mathbf{x}_{k}\right)}{\partial \mathbf{x}_{k}}
$$

\section{A. EKF for Dynamical Phase-Offset Estimation}

Here we consider the oversampled phase estimation scenario. So our state-space model is the one presented in Section II (eqs. 15,18).

As the state equation is linear we have directly that

$$
\frac{\partial f_{k-1}\left(\mathbf{x}_{k}\right)}{\partial \mathbf{x}_{k}}=\mathbf{M}
$$

The phase noise covariance $\mathbf{Q}$ is independent from $k$ and has only two non-zero elements, namely

$$
[\mathbf{Q}]_{1,1}=\frac{\sigma_{w}^{2}}{S} ;[\mathbf{Q}]_{3,3}=\sigma_{n}^{2}
$$

As we introduced the coloured noise $b_{k}$ into the state, there is no observation noise and the covariance matrix $\mathbf{R}$ is null. The observation equation is non-linear with the state, so we have to apply a linearization. We define

$$
\mathbf{g}=\frac{\partial g_{k}\left(\widehat{\mathbf{x}}_{k \mid k-1}\right)}{\partial \mathbf{x}_{k}}=\left[\begin{array}{lllll}
i A_{k} e^{i \widehat{\theta}_{k \mid k-1}} & 1 & 0 & \cdots & 0
\end{array}\right]^{T}
$$

Replacing these expressions into the general EKF expressions ( [5]) leads to the oversampled algorithm:

$$
\begin{cases}\mathbf{P}_{k \mid k-1} & =\mathbf{M} \mathbf{P}_{k-1 \mid k-1} \mathbf{M}^{H}+\mathbf{Q}_{k-1} \\ \widehat{\mathbf{x}}_{k \mid k-1} & =\mathbf{M} \widehat{\mathbf{x}}_{k-1 \mid k-1} \\ \mathbf{K}_{k} & =\mathbf{P}_{k \mid k-1} \mathbf{g}^{H}\left\{\mathbf{g} \mathbf{P}_{k \mid k-1} \mathbf{g}^{H}\right\}^{-1} \\ \mathbf{P}_{k \mid k} & =\left[\mathbf{I}-\mathbf{K}_{k} \mathbf{g}\right] \mathbf{P}_{k \mid k-1} \\ \widehat{\mathbf{x}}_{k \mid k} & =\widehat{\mathbf{x}}_{k \mid k-1}+\mathbf{K}_{k}\left[\mathbf{y}_{k}-A_{k} e^{\left.i \widehat{\theta}_{k \mid k-1}-\widehat{b}_{k \mid k-1}\right]}\right.\end{cases}
$$

\section{BAYESIAN CRAMÉR-RAO BOUND}

In this section we recall the expression of the on-line Bayesian Cramér-Rao Bound (BCRB) for an oversampled phase estimation problem [7]. This bound is particularly suited for problems where an a priori information is available [8].

The BCRB matrix can be written as

$$
\mathbf{B C R B}=\{\mathbf{B}\}^{-1}=\left\{\mathbf{B}^{D}+\mathbf{B}^{P}\right\}^{-1}
$$

We have to compute two terms. The first one, $\mathbf{B}^{D}$, represents the average information about $\boldsymbol{\theta}$ brought by the observations $\mathbf{y}$

$$
\left[\mathbf{B}^{D}\right]_{k, l}=2 \Re\left\{A_{l}^{*} A_{k}\left[\boldsymbol{\Gamma}^{-1}\right]_{k, l} e^{\Psi}\right\}
$$

where

$$
\Psi=\left\{-\frac{1}{2}\left(\left[\boldsymbol{\Sigma}^{-1}\right]_{k, k}+\left[\boldsymbol{\Sigma}^{-1}\right]_{l, l}-2\left[\boldsymbol{\Sigma}^{-1}\right]_{k, l}\right)\right\}
$$



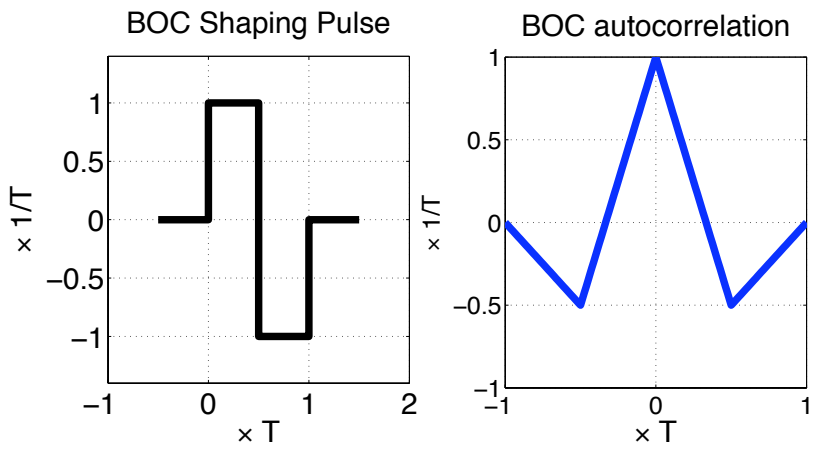

Fig. 1. BOC shaping function $\Pi(t)$ and its autocorrelation $g(t)$

The second term, $\mathbf{B}^{P}$, represents the information available from the prior knowledge on $\boldsymbol{\theta}$,

$$
\mathbf{B}^{P}=\frac{1}{\sigma_{w}^{2} / S}\left(\begin{array}{ccccc}
1 & -1 & 0 & \cdots & 0 \\
-1 & 2 & -1 & \ddots & \vdots \\
0 & \ddots & \ddots & \ddots & 0 \\
\vdots & & -1 & 2 & -1 \\
0 & \ldots & 0 & -1 & 1
\end{array}\right)
$$

In the general case, the on-line BCRB associated to observation vector $\mathbf{y}=\left[y_{1} \cdots y_{N}\right]$ is equal to entry $(N, N)$ of the BCRB matrix, $[\mathbf{B C R B}]_{N, N}$.

As we analyse the estimation problem in a DA scenario the bound depends on the transmitted sequence a. In this paper we suppose the transmission of a known sequence to analyse the performance of the proposed algorithm and the bound. We note that, contrary to [7] where the proposed bound was the minimum over a set of sequences, the BCRB is computed in this paper over the transmitted known sequence.

When $S>1$ we have more than one sample/symbol. In this case we have different bounds depending on the position inside the symbol so the BCRB depends on the couple $(S, s)$. If we are interested in the optimal estimation values we set $s=0$. So the bound is obtained as

$$
B C R B(S, s)=[\mathbf{B C R B}(\mathbf{a})]_{N, N}
$$

with $N=(M-1) * S+s+1 . M$ corresponds to the number of transmitted symbols, so the length of the known sequence in our case.

\section{Discussion}

In this section we show the behaviour of the EKF by considering different scenarios. We assume the transmission over an AWGN channel of a M-sequences of length 511 bits, generated using a Linear Feedback Shift Register (LFSR) with characterisic polynomial $[1021]_{8}$ (octal representation). We consider three oversampling factors $(S=1,2$ and 4$)$ and a BOC shaping pulse (see figure 1). BOC shaping pulse is used in Galileo positioning system.

In the figures presented we plot the Mean Square Error (MSE) obtained by simulation versus the Signal to Noise Ratio

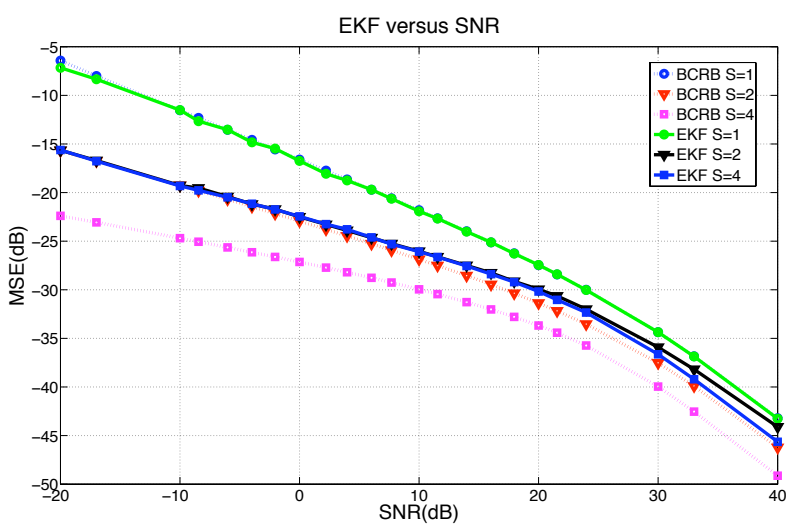

Fig. 2. EKF and BCRB versus the SNR for three different oversampling factors $S=1,2$ and 4, with a phase-noise variance $\sigma_{w}^{2}=0.001 \mathrm{rad}^{2}$.

(SNR). The SNR corresponds to the Carrier to Noise Ratio $\left(\frac{C}{N}\right)$ at the input of the receiver. In our case, as shaping pulse and symbols $a_{k}$ are normalised (i.e $\sigma_{a}^{2}=1 ; g(0)=1$ ) this ratio is simply $\frac{C}{N}=\frac{1}{\sigma_{n}^{2}}$. We compute the MSE for the $T$ spaced optimal estimation values corresponding to the $s=0$ case for all oversampling factors $S$. We also give the on-line BCRB for each case as a reference.

Figure 2 and 3 superimpose versus the SNR, the on-line BCRB (see eq.(26)) and the EKF. For figure 2, we have a slow varying phase with variance $\sigma_{w}^{2}=0.001 \mathrm{rad}^{2}$ and for figure 3 , we have a phase with a fast evolution, $\sigma_{w}^{2}=0.01 \mathrm{rad}^{2}$. In both scenarios there's no offset from the optimal sampling instants, $\tau=0$.

One can see that for $S=1$ the performance of the EKF is the same as the theoretical result of the BCRB. For $S=$ 2 the performance of the algorithm are slightly looser when comparing to the bound. For $S=4$ we obtain the same or slightly better performance as in the $S=2$ case. In this case if we compare the algorithm with the bound we can see that the performance poorer. The gain increases with the oversampling factor $S$ and the interest of oversampling becomes clear at low SNR. The gain due to oversampling decreases as the SNR increases.

In figure 4 we analyse the EKF behaviour for a fixed SNR versus phase-noise variance. We present a scenario with a low $\mathrm{SNR}$ value, $\mathrm{SNR}=0 d B$. Here we can still measure the gain given by the oversampling and the good performance of the algorithm. The gain obtained with the oversampling is greater at weak $\sigma_{w}^{2}$. We also note that the performance of the algorithm at weak phase noise variance is really close from the bound. At very high $\sigma_{w}^{2}$ the performances become poorer compared to the bound. This is probably because for high SNR, the modeling error in the EKF linear approximation (see eq. 20) is not neglictible with respect to the noise level.

Figure 5 superimposes versus the SNR, the on-line BCRB and the EKF for a slow varying phase evolution scenario, $\sigma_{w}^{2}=0.001$, and a non-null offset $\tau=\frac{T}{8}$ for $S=1$ and $S=2$. We show in the same figure the performance of the EKF for a null offset $\tau=0$ as a reference. 


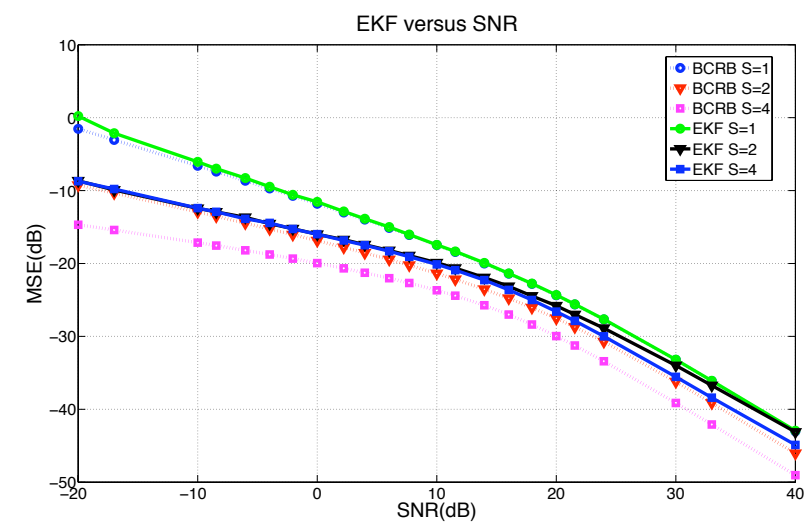

Fig. 3. EKF and BCRB versus the SNR for three different oversampling factors $S=1,2$ and 4 , with a phase-noise variance $\sigma_{w}^{2}=0.01 \mathrm{rad}^{2}$.

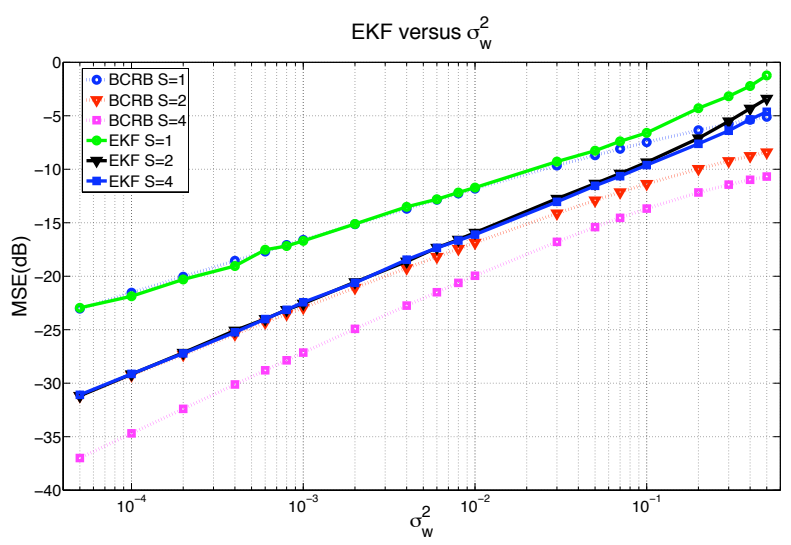

Fig. 4. EKF and BCRB versus the phase noise variance for three different oversampling factors $S=1,2$ and $4, \mathrm{SNR}=0 \mathrm{~dB}$.

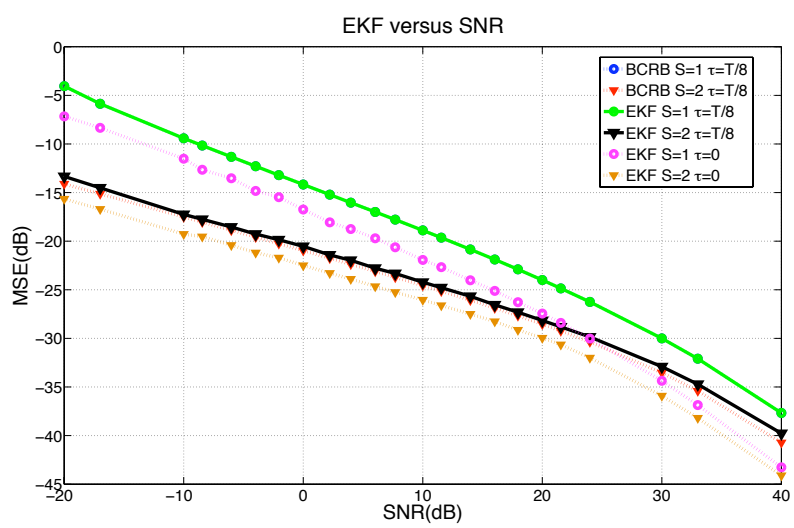

Fig. 5. EKF and BCRB in presence of a non nul offset for the sampling instants for two different oversampling factors $S=1$ and $2, \mathrm{SNR}=0 \mathrm{~dB}$.

We can see that the performances for both the bound and the algorithm are looser when having a non-null offset $\tau$. In general the performances decrease when increasing $\tau$ $\left(0 \leq \tau \leq \frac{T}{S}\right)$. We also note that the gain between different oversampling factors is greater at high SNR when having a non-null offset.

\section{CONCLUSION}

In this contribution, we have presented the Extended Kalman Filter for a realistic dynamical carrier phase estimation in an oversampled scenario. We have presented numerical results using a time limited pulse as done in satellite positioning systems. In such scenario, where the Shannon sampling theorem is not respected, we have shown the interest of using a fractionally-spaced method for phase estimation. The interest of using this algorithm with the oversampled signal becomes clear at low SNR. The results obtained with the EKF are close to the theoretical bound for slow and moderate varying phase evolutions.

\section{ACKNOWLEDGEMENT}

This work was supported in part by the French ANR (Agence Nationale de la Recherche), LURGA project.

\section{REFERENCES}

[1] H. L. Van Trees, Detection, Estimation and Modulation Theory. New York: Wiley, 1968, vol.1.

[2] S. M. Kay, Fundamentals of statistical signal processing: estimation theory. Upper Saddle River, NJ, USA: Prentice Hall, Inc., 1993.

[3] R. E. Kalman, " A new approach to linear filtering and prediction problems", J. Basic Eng., Trans. ASME, Series D, vol. 82, No.1, pp $35-45,1960$.

[4] R. E. Kalman and R. S. Bucy "New results in linear filtering and prediction theory",J. Basic Eng., Trans. ASME, Series D, vol. 83, No.3, pp. $95-108,1961$.

[5] B. D. O Anderson and J. B. Moore, Optimal Filtering. Englewood Cliffs, NJ, USA: Prentice Hall, 1979.

[6] A. H. Jazwinski, Stochastic Processes and Filtering Theory. Academic Press, Inc. , NY, 1970.

[7] J.Vilà Valls, J. M. Brossier and L. Ros, “ On-line bayesian Cramér-Rao bound for oversampled dynamical phase offset estimation", IEEE ISCCSP 2008, Malta, 12-14 March 2008.

[8] S. Bay, C. Herzet, J. M. Brossier, J. P. Barbot, and B. Geller, “ Analytic and Asymptotic Analysis of Bayesian Cramér-Rao Bound for Dynamical Phase Offset Estimation", IEEE Trans. Signal Processing, Jan. 2008.

[9] P. J. Kootsookos, " An extended kalman filter for demodulation of polynomial phase signals", IEEE Signal Processing Letters, Vol. 5, No. 3, March 1998.

[10] B. F. La Scala, R. R. Bitmead " Design of an extended kalman filter frequency tracker”, IEEE Signal Processing Letters, Vol. 5, No. 3, March 1998.

[11] A. Aghamohammadi, H. Meyr, G. Ascheid" Adaptive synchronization and channel parameter estimation using an extended kalman filter", IEEE Trans. on Communications, Vol. 37, No. 11, Nov. 1989.

[12] L.-L. Cheng, Z.-G. Cao "GPS Carrier phase measurement method based on data transition detection in high dynamic circumstance", Proc. WCCICCT, Vol.2, 21-25 Aug. 2000.

[13] P. O. Amblard, J. M. Brossier and E. Moisan, " Phase tracking: what do we gain from optimality?Particle filtering versus phase-locked loops", Elsevier Signal Processing, vol. 83, pp. 151-167, Oct. 2003. 